\title{
L'aventure libyenne et ses vécus politiques et sécuritaires pour les migrants maliens
}

Almamy Sylla

\section{OpenEdition}

\section{Journals}

Édition électronique

URL : https://journals.openedition.org/anthropodev/1012

DOI : 10.4000/anthropodev. 1012

ISSN : 2553-1719

Éditeur

Presses universitaires de Louvain

Édition imprimée

Date de publication : 1 décembre 2020

Pagination : 137-153

ISBN : 978-2-39061-078-6

ISSN : 2276-2019

Référence électronique

Almamy Sylla, "L'aventure libyenne et ses vécus politiques et sécuritaires pour les migrants maliens », Anthropologie \& développement [En ligne], 51 | 2020, mis en ligne le 01 juin 2021, consulté le 31 janvier 2022. URL : http://journals.openedition.org/anthropodev/1012 ; DOl : https://doi.org/10.4000/ anthropodev. 1012

La revue Anthropologie \& développement est mise à disposition selon les termes de la Licence Creative Commons Attribution 4.0 International. 


\title{
L'aventure libyenne et ses vécus politiques et sécuritaires pour les migrants maliens
}

\begin{abstract}
Almamy Sylla ${ }^{1}$
Cet article décrit et analyse I'historicité des expulsions des Maliens de Libye, à partir de la seconde moitié des années 1980, et l'instrumentalisation des enjeux migratoires dans les politiques économiques et sécuritaires nationales et interrégionales à partir des perceptions et des perspectives des rapatriés. Il démontre comment la fabrique des rapatriés, une catégorie de migrants de retour au pays, participe à la complexité de la situation contemporaine au Mali. Il souligne aussi comment, au tournant des années 2000, I'Union européenne a contribué à renforcer ce processus. L'article s'appuie sur des données issues d'entretiens réalisés avec des rapatriés maliens de Libye et avec des officiels maliens impliqués dans la gestion des migrations, ainsi que sur des débats radiophoniques et des conférences de presse sur le rapatriement et la crise migratoire en Libye. L'article contribue aux débats sur les parcours des migrants, souvent marqués par la violence, l'insécurité, la stigmatisation et l'exclusion.

This article describes and analyzes the historicity of the evictions of Malians from Libya from the second half of the 1980s and the instrumentalization of migration in national and interregional economic and security policies from the perceptions and perspectives of repatriates. It demonstrates how the complexity of the contemporary situation in Mali owes much to the historical depth of the making of the returnees, a category of return migrants, which from the 2000s onwards was reinforced by the European Union. The article uses interview data with returnees from Libya, Malian officials in charge of migration management, and radio debates and press conferences on the migration crisis, border management policies and the presence of migrant workers in Libya. Finally, it intends to contribute to the debates on migrants' pathways, often marked by violence, insecurity, stigma and exclusion.
\end{abstract}

\footnotetext{
${ }^{1}$ Enseignant-chercheur, université des lettres et des sciences humaines de Bamako (ULSHB), laboratoire d'anthropologie comparative, engagée et transnationale (LACET) ; syllaalmamy@gmail.com
} 


\section{Introduction}

Maintenant, c'est devenu difficile de stopper l'immigration. C'est une menace très sérieuse. Nous sommes prêts à intervenir, à servir d'intermédiaire. (extrait du discours de Kadhafi, 11 juin 2009) ${ }^{2}$

Par ces mots, Kadhafi s'exprimait sur l'accord qu'il venait de parachever à Rome, le 11 juin 2009, avec l'Italie, sur la gestion de la migration. L'accord en question faisait partie d'une série d'accords conclus entre la Libye et l'Union européenne (UE) dont l'objectif était de donner un cadre juridique d'intervention afin de réduire, voire de décourager, l'immigration des travailleurs immigrés dits clandestins en provenance du continent africain. Ce cadre d'intervention et de collaboration fondait ses arguments sur un imaginaire criminalisant lié au mythe de l'invasion de l'UE par les migrants originaires d'Afrique subsaharienne, perçus comme de potentielles menaces de déstabilisation sociale et économique pour l'UE (Rousseau et Foxen, 2006 ; Valluy, 2006 ; De Haas, 2007). En partant de ce cadre euro-africain de gestion des migrations, cet article analyse les expulsions de Maliens de Libye et leur prise en charge politique, à partir de la seconde moitié des années 1980, la production de la catégorie de rapatriés et l'instrumentalisation des enjeux migratoires dans les politiques économiques et sécuritaires nationales et interrégionales à partir des perceptions et perspectives des rapatriés ${ }^{3}$ de Libye au Mali. L'expulsion, "l'illégalisation » des migrants (De Genova, 2017) et la subalternisation de certains emplois sont devenues des modes de gestion des enjeux migratoires et sécuritaires en Libye et au Mali.

Dans cet article, je traite d'une part des politiques de gestion des migrations en Libye telles qu'elles sont vécues et racontées par les rapatriés maliens, avec leurs stratégies d'adaptation; et d'autre part de la gestion par les pouvoirs publics maliens des rapatriés en fonction du pendant sécuritaire et du "potentiel de menace " dont ils seraient porteurs tant en Libye qu'au Mali. L'article s'appuie sur des matériaux empiriques issus de mes recherches doctorales sur les rapatriés au Mali. En m'inscrivant dans une approche socio-anthropologique, mon corpus de données est constitué d'entretiens avec des rapatriés, d'observations de leurs pratiques de mobilisation politique, de matériaux radiophoniques traitant des migrations subsahariennes en Libye, d'articles de journaux et d'entretiens avec des officiels maliens et des acteurs de la société civile malienne impliqués dans les enjeux migratoires. L'article se situe à l'intersection des études des politiques migratoires, des perceptions des rapatriés de leurs expériences migratoires en Libye, et de la façon dont l'État malien a pris en charge leurs rapatriements.

\footnotetext{
${ }^{2}$ Cité dans le film documentaire Le piège (Dereims, 2011).

${ }^{3}$ Légalement, le "rapatrié » est un ressortissant dont le pays d'origine a assuré légalement le retour sur son territoire, généralement suite à une situation de crise ou de guerre. Mais, au Mali, comme dans d'autres pays subsahariens, il en découle une catégorie émique de "rapatriés ». Cette dernière est plus large que celle strictement juridique et englobe l'ensemble des Maliens de retour au Mali après un séjour en Libye, que leur retour ait ou non été pris en charge par l'État malien. C'est cette terminologie émique qui sera utilisée tout au long de cet article.
} 
Je présente tout d'abord la contextualisation historique du nexus migration, crises et sécurité entre le Mali et la Libye. Les contextes historiques de commerce transsaharien, de pèlerinage, de rébellions et de sècheresse au Sahel expliquent la présence en assez grand nombre de migrants maliens en Libye. En vue de formaliser et de réguler cette présence, une convention règlementant l'utilisation de la main-d'œuvre malienne par la Libye est signée en décembre 1980. À la suite de la signature de cette convention, on assiste à des vagues d'expulsion de Maliens depuis la Libye. En deuxième lieu, j'aborde les vécus de la crise libyenne et le rapatriement. La mise en place des opérations de rapatriement et les vécus des rapatriés de la crise libyenne sont traités de manière spécifique dans cette section. Ensuite, il est traité des perceptions des rapatriés de l'instrumentalisation libyenne des migrants. Enfin, la politisation du retour et la construction d'identités plurielles à partir du retour de la Libye sont abordées. La pluralité des prises en charge des rapatriés de Libye au Mali a donné lieu à des modes d'actions collectives, à des accusations de collusion de l'État du Mali avec les rapatriés armés et à l'émergence d'identités particulières.

\section{Contextualisation historique du nexus migration, crises et sécurité entre le Mali et la Libye}

Le Mali est un pays historiquement marqué par les migrations. II se situe au carrefour de plusieurs routes et réseaux migratoires vers les pays africains dont la Libye. Les mobilités maliennes vers l'Afrique du Nord ont comme toile de fond les mobilités religieuses pour le pèlerinage et la formation (Brachet et al., 2011 ; Bava et Boissevain, 2014) ainsi que le commerce transsaharien (Grégoire et Schmitz, 2000 ; Lovejoy, 2011). S'appuyant sur ce continuum historique, la Libye est devenue l'une des destinations les plus privilégiées par les Maliens dans la période postcoloniale. Cet intérêt s'explique aussi par l'entrée en récession économique de la Côte d'Ivoire et du Nigeria dans les années 1980 (De Haas, 2007; Adepoju, 2009; Sylla, 2019); les sècheresses au Sahel des années 1970-1980 (Brachet, 2009 : 112 ; Pliez, 2000 ; Bredeloup et Zongo, 2005 ; De Haas, 2007) ; ou encore l'exploitation du pétrole et la réalisation de travaux d'infrastructures socio-économiques en Libye avec un besoin important de main-d'œuvre (Brachet, 2009 ; Hamood, 2006 ; Andersson, 2014). Si la Libye a incité une migration subsaharienne pour ses besoins économiques grandissants (Bredeloup et Pliez, 2005 ; Mounkaïla, 2015), elle a également procédé à des vagues d'expulsion répétées de migrants subsahariens. Ainsi, dans les années 1980, considérées comme l'apogée des migrations maliennes en Libye, cette dernière a simultanément expulsé environ 11000 migrants maliens entre 1985 et 1986 (Kanté et Camara, 1987). 


\section{Les années 1980-1990 en Libye : la convention d'emploi de la main-d'œuvre d'origine malienne et la fabrique des expulsables maliens}

Le 12 décembre 1980, la Libye et le Mali signaient une convention qui régulait l'emploi de la main-d'œuvre d'origine malienne en Libye ${ }^{4}$. Cette convention devait mettre fin aux vagues de migrations dites "irrégulières" et, selon la rhétorique officielle, offrir de bonnes conditions de travail aux migrants maliens recrutés par les filières officielles. Ses articles 2, 4, 5 et 11 obligeaient les candidats maliens à l'émigration en Libye à passer par l'Office malien de main-d'œuvre (OMO) pour négocier des contrats de travail. Cette procédure administrative était perçue comme relativement lourde, longue, coûteuse et compliquée par ces migrants qui, pour la plupart, aspiraient à plus de liberté. De plus, comme le soulignent Sylvie Bredeloup et Mahamadou Zongo (2005: 125) dans leurs travaux sur les rapatriés burkinabés, "le certificat médical rebaptisé "ticket sanitaire" était exigé pour les migrants subsahariens » à destination de la Libye. L'instauration de ce certificat médical faisait suite à une forme de "pathologisation " des Subsahariens amplifiée "par la médiatisation du VIH, de sa diffusion en Afrique noire, ou encore de l'épidémie d'Ebola, et qui ont contribué à ancrer l'idée que les Noirs étaient des porteurs de maladies graves" (El Miri, 2018 : 110). Ce processus bureaucratique était aussi vécu comme une entorse à une culture de migration qui fonctionnait suivant des réseaux familiaux, communautaires et d'amitié (Brachet, 2009 ; El Miri, 2018). À titre d'exemple, voici comment cette convention était perçue par ce rapatrié malien parti en Libye en 1986 sans emprunter les voies légales:

J'ai quitté Bamako précisément le 20 janvier 1986 pour la Libye après plusieurs tentatives infructueuses. En ce temps, il fallait d'abord déposer une caution de 150000 francs maliens avant de demander le visa. Comme je ne me retrouvais pas dans ces schémas, j'ai dû partir [...] par étapes. (extrait d'entretien d'un rapatrié malien responsable de I'AMARLIM, janvier 2013, Bamako)

Malgré les voies légales ouvertes par cette convention de 1980, la migration «par étapes " dont parle mon interlocuteur était la voie la plus empruntée par les Maliens souhaitant se rendre en Libye. Cette pratique migratoire était principalement motivée par une situation économique difficile, les difficultés d'application de la convention et une volonté d'échapper au contrôle étatique du régime dictatorial ${ }^{5}$ de Moussa Traoré. Rappelons que, sous la première république de Modibo Keïta, l'opposition des pouvoirs publics à l'exode et à l'émigration avait enclenché un sentiment de méfiance par rapport à l'État chez les Maliens envisageant les voies de la migration (Tounkara, 2003 ; Keita, 2009 : 222-223).

Avec cette convention Mali-Libye de décembre 1980, malgré les difficultés de son application imputées à l'ambivalence des relations entre les deux États, il existait une

\footnotetext{
${ }^{4}$ Convention relative à l'emploi de la main-d'œuvre entre la Jamahiya arabe libyenne populaire et socialiste et la république du Mali, 12 décembre 1980, Bamako.

${ }^{5}$ De novembre 1968 à mars 1991, le Mali vit sous un régime dictatorial avec pour corollaire les atteintes aux libertés individuelles, la répression, la fuite et l’exil des opposants au régime.
} 
" volonté politique d'ouverture de la Libye » (entretien avec le conseiller du ministère des Maliens de l'extérieur et de l'Intégration africaine (MMEIA), janvier 2013). Cette volonté d'ouverture s'inscrivait au nom de la "réalisation de l'Unité africaine ", adressée à l'ensemble de l'Afrique subsaharienne (De Haas, 2007). Dans ses relations bilatérales avec le Mali, la Libye intensifia ses investissements économiques et son aide au développement avec, par exemple, des vivres et la télévision publique offerts au Mali en 1983. Cette ouverture était couplée à la fermeture des frontières aux travailleurs maliens dits "illégaux », fermeture dont les expulsions étaient l'expression la plus illustrative. Ce paradoxe de la politique extérieure libyenne est perçu ainsi par ce leader associatif ayant migré en Libye en 1983 : c'est comme si « la Libye donnait quelque chose avec la main droite et la reprenait de suite avec la main gauche " (entretien avec un rapatrié et porteparole de l'AMARLIM, juin 2012).

Par ailleurs, la période d'application de cette convention coïncida avec une conjoncture économique ${ }^{6}$ difficile pour la Libye, due aux sanctions économiques des USA dès 1982 et à I'imposition par l'ONU d'un embargo aérien et sur les armes en 1992. Avec la crise économique qui en découla, on assista à la production d'une catégorie de migrants dits "illégaux » et expulsables (Beaugé et Burgat, 1986). Ainsi, au lieu de favoriser la mise en place de filières d'une migration légale grâce à cette convention, les expulsions, depuis la Libye, de migrants subsahariens en général et maliens (Kanté et Camara, 1987), burkinabés et nigériens en particulier devinrent importantes entre 1985 et 1995 . Pour la Libye, il s'agissait officiellement " de contingenter les migrations de travail » (Pliez, 2004: 10). Cette décision est intervenue dans un contexte où le modèle rentier libyen, fondé sur des contrats internationaux régissant la venue des travailleurs migrants, s'est effondré avec la chute du cours des barils de pétrole (Pliez, 2004). Une majeure partie des migrants subsahariens dont la présence était tolérée en Libye au nom de l'idéal panafricain devenaient de fait des "illégaux " " (De Genova, 2017 ; Scheel, 2017 ; Agier, 2008) et donc expulsables. Cette situation ne découlait pas des clauses des conventions ni des contrats régissant la présence des migrants subsahariens ${ }^{8}$, mais bien du fait du contexte géopolitique et économique complexe de la Libye. Face à l'ampleur du phénomène des expulsions de migrants maliens à cette époque, la revue Jamana consacra un numéro spécial en 1987 sur les tragédies et les traitements inhumains vécus par ces travailleurs migrants en Libye. Un ancien conseiller technique au MMEIA notait aussi :

[...] la Libye a fixé des conditions pour l'entrée et le séjour sur son territoire. [...] Nos ressortissants ne se mettant pas en règle pour y rentrer [...] il devenait difficile pour eux d'avoir des papiers et, en conséquence, ils travaillaient au noir. (extrait d'entretien avec un ancien conseiller technique au MMEIA, janvier 2013, Bamako)

\footnotetext{
${ }^{6}$ Les revenus des exportations pétrolières sont passés de 20 milliards de dollars en 1981 à 5 milliards en 1986 (Bensaâd, 2012 : 89).

${ }^{7}$ En 1987, 80 \% des Maliens vivant en Libye étaient des clandestins, donc en situation irrégulière (Kanté et Camara, 1987 : 17).

${ }^{8}$ Il existait en Libye un « vide juridique » concernant les droits d'entrée et de séjour (Bredeloup et Zongo, 2005 : 125).
} 
Le récit ci-dessous apporte d'autres éclairages sur les processus "d'illégalisation » (De Genova, 2017) des migrants maliens en Libye, enclenchés à cette époque et en cours jusqu'en 2010 :

[...] un Libyen policier t'interpelle, même si tu as le passeport avec le cachet du visa, s'il décide de te le prendre, il n'y a pas de " médicament " [remède]. II te dit que c'est la Libye qui t'a accordé le visa, on le déchire pour te ramener chez toi... ou en prison. (entretien avec une rapatriée ayant vécu en Libye de 2005 à 2011, février 2013, Bamako)

L'autre versant de la production de migrants « irréguliers » était le recours au travail au noir et la subalternisation des niches économiques pour les migrants dits "irréguliers » (Pliez, 2004 : 144). Selon les rapatriés rencontrés et comme le soulignent M. Kanté et A. Camara (1987), le travail au noir réalisé par ces migrants dits « irréguliers » concernait le travail journalier informel et les niches professionnelles ne demandant pas de qualification particulière. Un rapatrié me raconte :

[en Libye] au foyer, le matin, tout le monde sort. Le matériel de travail, c'est le burin et le marteau. Le burin et le marteau, pourquoi ? C'est pour les travaux difficiles comme casser un mur, creuser un trou. (entretien avec un rapatrié de la Libye, octobre 2015, Bamako)

Après les expulsions de masse de 1985 et 1986, la Libye se dota d'un arsenal juridique règlementant les conditions d'entrée et de séjour sur son territoire (De Haas, 2007 ; Bartolomeo et al., 2011). Les années 1990 se sont inscrites dans la continuité des dynamiques observées ci-dessus. La Libye oscillait entre appel à la main-d'œuvre, panafricanisme et expulsions (De Haas, 2007; Pliez, 2002). La politique libyenne d'ouverture permit néanmoins aux migrants subsahariens, dont les Maliens, de circuler librement dans le cadre de la CEN-SAD ${ }^{9}$, en s'appuyant sur une rhétorique universaliste, anticolonialiste et révolutionnaire dont la finalité était "de se servir de la question migratoire comme d'une "arme diplomatique" de positionnement dans les mondes arabe, africain et européen » (Puig, 2019 : 1). Ali Bensaâd (2012: 91) analyse ces manœuvres diplomatiques libyennes comme une "stratégie de déverrouillage " de l'embargo. Cependant, les difficultés économiques résultant de ce dernier entrainèrent une réduction de la main-d'œuvre étrangère. C'est dans ces circonstances que, « en juillet 1995, le Congrès général du peuple dénonce l'immigration clandestine qui véhicule selon lui le crime organisé et le trafic de drogue. L'annonce est suivie du départ de 335000 étrangers, dont 200000 expulsés » (Perrin, 2009 : 292-293).

\section{L'après-embargo onusien}

À partir de 1999, les sanctions américaines et du Conseil de sécurité des Nations unies contre la Libye furent progressivement levées. Cette période continua d'être entrecoupée d'expulsions mais permit aussi l'installation de travailleurs migrants du fait d'une grande

\footnotetext{
${ }^{9}$ La Communauté des États sahélo-sahariens (CEN-SAD) est créée en février 1998 initialement autour du Tchad, du Burkina Faso, du Mali, du Niger et de la Libye.
} 
absorption de la main-d'œuvre immigrée rendue possible par le nouvel essor économique libyen. Un migrant malien ayant séjourné en Libye dans la région de Sebbah, puis à Tripoli, se réjouissait de ses conditions d'existence à cette époque :

Regarde ces photos, surtout celle-là. C'est moi et ma famille en Libye. Tu m'as vu ici en personne, non? Je suis quelqu'un qui a du gabarit. Regarde-moi comme je suis aujourd'hui. Tu ne vois pas la misère en moi ? (entretien avec un rapatrié ayant séjourné pendant 29 ans en Libye, août 2012, Bamako)

Toutefois, l'amélioration des conditions d'existence offertes aux travailleurs migrants était mal perçue par des Libyens dont le leitmotiv était " Libyans first " (Bensaâd, 2002, 2012). En septembre 2000, des émeutes se soldèrent par la mort de 130 migrants subsahariens (Pliez, 2004). Ainsi, pour atténuer les tensions au sein de la population libyenne, les autorités du pays prirent des mesures renforcées à l'encontre des migrants subsahariens: restriction de l'immigration illégale, détention prolongée et arbitraire ${ }^{10}$, violence physique et expulsion (De Haas, 2007; Haddad, 2007). À la suite de ces manifestations, Bredeloup et Zongo (2005 : 127) rapportent que, « en juillet 2004, les 26 ambassades africaines présentes à Tripoli ont été informées du projet de rapatrier dans leurs pays respectifs tous ceux qui étaient entrés illégalement sur le territoire libyen ». C'est ainsi qu'entre 2002 et 2008 plus de 2670 Maliens furent expulsés de la Libye vers le Mali (Ballo, 2009). Cette situation coïncidait aussi avec l'acceptation de la Libye de « jouer le rôle de "sentinelle avancée" pour les pays de l'UE principalement préoccupés par la défense de leur "forteresse" nouvellement élargie » (Bredeloup et Zongo, 2005 : 124).

Face à la gravité des expulsions et à leurs répercussions sur les conditions de vie des expulsés au Mali, Oumar Mariko, député à l'Assemblée nationale du Mali siégeant dans l'opposition, interpella le ministre des Maliens de l'extérieur de l'époque sur "les expulsions répétitives de Maliens et les relations ambiguës que l'État malien entretenait avec les États libyen, français et espagnol sur les questions d'intégration africaine et des droits de l'Homme des migrants " (questions orales à l'Assemblée nationale du Mali, 2 décembre 2008). Cette prise de position de Mariko pour la défense des droits des migrants en Libye fut appréciée par un rapatrié à Kita ayant vécu ces événements :

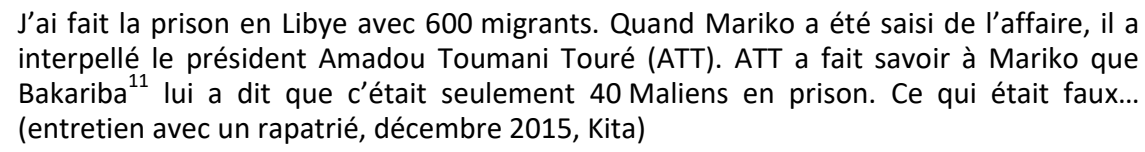

Dans le même sillage, un collectif d'expulsés de Libye a tenu une conférence de presse à Bamako en mai 2010 pour dénoncer le caractère arbitraire des expulsions et des emprisonnements de migrants en Libye :

\footnotetext{
${ }^{10}$ Selon les estimations d'Amnesty International (2017: 8), 20000 refugiés et migrants étaient enfermés dans les centres de détention en 2012 en Libye.

${ }^{11}$ Les migrants maliens se servaient de ce sobriquet pour designer Kadhafi par peur de représailles en le désignant par son vrai nom.
} 
Nous dénonçons le régime policier libyen qui s'est totalement transformé en gendarme des frontières de l'Europe. Nous dénonçons également les politiques xénophobes et racistes (Frontex, directive retour, accords de réadmission...) de l'Union européenne qui sont sources de répression et d'inégalités. Nous informons l'opinion nationale malienne, africaine et internationale que plus de 10000 migrants sont détenus dans les prisons libyennes, et que si les bonnes volontés n'interviennent pas, ceux-ci vont mourir à petit feu. (communiqué de presse du Collectif de 149 Maliens expulsés de la Libye, 3 mai 2010)

À la suite de ces prises de position et de ces contestations, les expulsions de migrants depuis la Libye suscitèrent de virulents débats dans la presse malienne et au sein des organisations de la société civile. Dans la parution de L'Indicateur du Renouveau du 28 janvier 2011, Abdoulaye Diakité (2011) attirait l'attention de l'opinion nationale sur les traumatismes subis par les expulsés dus "à la violence de la répression et la nature du régime carcéral (privation de nourriture et d'assistance médicale pour les malades et les blessés), aux offenses à la dignité et la sensibilité humaines ». Au contraire, Le Soir de Bamako rapporte les propos de l'ambassadeur libyen à Bamako sur les expulsions, dénonçant leur amplification par les médias : « [...] il [le docteur Ali Mohamed Almagouri] a déploré les événements (de ladite expulsion) dont l'ampleur est surtout due aux médias [...] » (Diarra, 2008).

C'est dans cette atmosphère survoltée que survint le conflit armé en Libye en 2011, se soldant par des rapatriements volontaires d'environ 706000 migrants de 120 pays (Naik, 2012 : 1) dont environ 23000 Maliens (Sylla, 2019 : 156). Face à cette situation, diverses formes de prises en charge des travailleurs migrants ont été mises en œuvre en Libye et dans les pays tiers, comme les programmes de retour volontaire assisté par l'OIM et I'UNHCR, les comités ministériels ad hoc de rapatriement et d'accueil (Mounkaïla, 2015 : 100 ) et les projets d'aide à la réinsertion. C'est à la suite de ces opérations que les migrants maliens en Libye, de divers profils, ont été évacués vers le Mali à partir de mars 2011. À partir du conflit libyen, on a assisté à la production du rapatrié qui remplace celle de l'expulsé.

\section{Vécus de la crise libyenne, fuite et rapatriement}

Aux premières heures du conflit libyen, le 17 mars $2011^{12}$, une zone d'exclusion aérienne fut instaurée sur la Libye par le Conseil de sécurité des Nations unies. Cela entrava le rapatriement des ressortissants des États tiers par voie aérienne. Pour contourner cette difficulté, les représentations diplomatiques maliennes à Tripoli, à Tunis et au Caire, en coordination avec l'OIM et l'UNHCR, mirent en place un plan d'évacuation des Maliens en détresse. En fonction de l'occupation territoriale et des forces en présence en Libye, des Maliens sous contrôle du Conseil national de transition (CNT) $)^{13}$ à Benghazi furent évacués vers le camp de Salloum en Égypte, tandis que ceux dans les localités restées fidèles à

\footnotetext{
${ }^{12}$ La mesure a été assouplie à partir de novembre 2011.

${ }^{13}$ Le CNT est une autorité politique de transition instituée le 27 février 2011 dans les différentes villes de Libye tombées aux mains des insurgés pour conduire le combat contre le régime dirigé par Kadhafi.
} 
Kadhafi furent évacués sur le camp de Choucha en Tunisie. Environ 80 rotations d'avions assurèrent le rapatriement des Maliens à partir de ces deux camps (entretien avec un agent de la Protection civile, janvier 2014, Bamako). Une troisième voie s'ouvrit par le Niger et le Tchad pour des migrants maliens bloqués dans le Sud libyen (Sebbah). Ces derniers regagnèrent ensuite Gao, où leur acheminement vers Bamako fut organisé par les services déconcentrés de l'État du Mali, la Croix-Rouge, la Maison des migrants et l'association des transporteurs de Gao. Si les opérations d'évacuation étaient coordonnées par l'OIM et I'UNHCR, la phase d'identification et de délivrance des laissez-passer était coordonnée par les ambassades du Mali à Tripoli, au Caire, à Tunis et par le Conseil de base des Maliens en Libye. Un rapatrié ayant rejoint la Libye en 2001 à partir d'Al Barakat (Ghadamès), ex-cuisinier à l'ambassade d'Argentine à Tripoli, raconte ici l'euphorie de son retour au Mali le 28 avril 2011 :

Quand l'OIM nous accueillait [en Tunisie], je ne savais pas qu'aujourd'hui allait arriver. La première chose qu'ils nous demandaient était qui veut téléphoner au pays ? C'était gratuit, le manger était gratuit. (entretien avec un rapatrié de la Libye de 40 ans, septembre 2012, Bamako)

Une autre rapatriée d'avril 2011 donne plus de détails sur les conditions et l'organisation de son retour au Mali :

Quand ça n'allait pas, un matin je suis allée à l'ambassade. J'ai pris un laissez-passer à 10 dinars et déposé une photocopie de ma pièce d'identité. [...] un minibus assurait le transport à la frontière tuniso-libyenne à 50 dinars par passager. Au poste frontalier, on payait quelque chose aussi... C'est là qu'on nous donnait à manger ; les médecins venaient nous consulter. Sur place, le consul du Mali à Tunis organisait le rapatriement. (entretien avec une rapatriée de Libye, janvier 2013, Bamako)

La question du laissez-passer, introduite ci-dessus, était devenue une controverse entre les candidats au retour et les services diplomatiques maliens à Tripoli. Au début du conflit, il était délivré gratuitement pour les ressortissants maliens souhaitant retourner au pays. Il fit ensuite l'objet de spéculation, d'après plusieurs rapatriés, lorsque le conflit devint violent. Ainsi, le laissez-passer était délivré contre une demande manuscrite timbrée, une copie d'une pièce d'identité et un timbre fiscal de 10 dinars. D'après plusieurs rapatriés, en devenant plus nombreuses, les demandes débouchèrent sur des pratiques informelles pour obtenir un laissez-passer. La question du laissez-passer est devenue incontournable pour les émigrés maliens en Libye qui n'avaient pas de passeport valide ni de carte consulaire. Lorsqu'ils se sont trouvés pris au piège de la guerre, le seul moyen pour regagner le Mali était le laissez-passer.

Une fois les émigrés arrivés au Mali, la réponse nationale se mit en route. Un comité interministériel de coordination des opérations de rapatriements élargi aux partenaires sociaux (protection civile, ONG, organisations humanitaires et religieuses, associations de migrants et municipalité de Bamako) était chargé de l'accueil, la documentation, l'assistance, la restauration, le transport et l'hébergement des rapatriés pour une période de 72 heures. Face à l'épreuve du retour (déconnexion avec les structures sociales, situation politique et économique difficile au Mali, manque de programmes d'aide à la réinsertion, difficultés d'hébergement après les 72 heures, etc.), en avril 2011, un groupe 
de rapatriés mit en place une association dénommée Association des Maliens rapatriés de la Libye et du Maghreb (AMARLIM) pour dénoncer les conditions d'accueil, défendre leurs droits à la réinsertion et revendiquer des biens restés en Libye ou dans les pays de transit. Parmi eux, il y avait des travailleurs migrants installés en Libye de longue date, des migrants de transit, d'anciens membres de la légion islamique et des mercenaires. Parmi ces rapatriés, certains furent catégorisés comme d'anciens "rebelles ", des rapatriés armés et des mercenaires. Cela leur valut souvent des prises en charge particulières par l’État malien.

\section{Instrumentalisation libyenne des migrants maliens et leurs perceptions}

Quand le conflit libyen commença en février 2011, le régime de Kadhafi et le CNT utilisèrent la rente migratoire comme moyens de pression et de séduction à destination de I'UE. Le régime de Kadhafi menaçait de cesser sa coopération dans la lutte contre l'immigration clandestine, tandis que le CNT se précipita pour conclure des accords avec I'Italie sur la maîtrise de l'immigration et le contrôle des frontières (Bensaâd, 2012 : 84 ; Rihouay, 2012).

Par ailleurs, plusieurs jeunes rapatriés à Bamako en juin 2012, qui avaient échoué à traverser la Méditerranée, projetaient de retourner en Libye. Ils souffraient de leurs difficultés de réinsertion au Mali tandis que leur parvenaient les échos de conditions "favorables» de traversée de la Méditerranée. D’autres rapatriés à Bamako entrevoyaient l'avènement du nouvel ordre politique comme une opportunité historique pour obtenir un emploi en Libye :

Nous avons décidé de revenir chez nous pour être respectés. Mais ça a été la pire des choses. Le devoir de l'État est de nous trouver un travail. S'il ne le peut pas, [...] moi j'ai un contrat illimité avec mon employeur libyen qui est sous leurs ordres [du CNT] en Libye... Je peux y retourner pour travailler... (entretien avec un rapatrié de la Libye, février 2013, Bamako)

Au Mali, le retour des rapatriés de Libye s'est fait dans un contexte social « marqué par un mécontentement généralisé ", des accusations de " corruption effrénée dans l'appareil d'État et la hiérarchie militaire " et de supposée "collusion entre le régime et les rebelles » (Tounkara, 2013 : 65-66). Déçus des conditions d'accueil et des offres de réinsertion au Mali, des rapatriés envisageaient un retour en Libye sur base des « bonnes relations " et des " bons souvenirs " qu'ils avaient entretenus avec des Libyens comme, par exemple, leurs employeurs. Ainsi, un rapatrié raconte l'appui et la protection qu'il a reçus de son patron aux temps forts du conflit libyen: "Mon patron m'avait dit qu'il mourra là où je mourrai. De ce fait, aller à la boutique ne m’était pas obligatoire » (entretien avec un rapatrié de la Libye, septembre 2015, Bamako).

Ayant rejoint la Libye après son échec au baccalauréat en 2001, ce rapatrié raconte sa perception de l'instrumentalisation des migrants maliens dans le conflit libyen au gré du positionnement du Mali dans celui-ci : 
On y était resté en attendant Dieu... La situation d'un ami d'enfance en prison m'intriguait beaucoup... II était accusé « d'intelligence » avec les forces pro-Kadhafi. (entretien avec un rapatrié de la Libye, octobre 2015, Bamako)

Le recrutement et la présence de combattants maliens sur le sol libyen mentionnés par cet interlocuteur ravivèrent des discussions autour de la convention de 1980 sur l'emploi de la main-d'œuvre malienne à destination de la Libye. D'après un conseiller technique au MMEIA, en 2011, une délégation libyenne s'adressa au Mali afin de réactiver cette convention obsolète.

Quand le conflit a éclaté, c'est en ce moment-là qu'une délégation libyenne est venue pour réactiver cet accord-là en arguant qu'ils avaient besoin de travailleurs. Nous avions compris que c'était pour les engager dans le conflit... (entretien avec un conseiller technique au MMEIA, janvier 2013, Bamako)

Le gouvernement malien n'a pas émis un avis favorable en 2011 à la demande libyenne de recruter de la main-d'œuvre malienne dans le cadre de la convention de décembre 1980, par crainte de légaliser le recrutement de mercenaires maliens sous le couvert d'une convention officielle (entretien avec un conseiller technique au MMEIA, janvier 2013, Bamako). Malgré cette fin de non-recevoir, des rapatriés et la presse rapportèrent que des Maliens auraient rejoint la Libye par des filières officieuses de recrutement (Lohmann, 2011 : 7 ; Le Tallec, 2011).

À l'aéroport de Mitiga, quand nous attendions notre embarquement, un bus suivi de la voiture de l'ambassadeur du Mali à Tripoli conduisait des prisonniers de guerre menottés. On leur a enlevé les menottes sous nos yeux. C'est dans les toilettes de l'aéroport que certains se sont lavés et se sont rhabillés. (entretien avec un rapatrié de la Libye, décembre 2011)

Cependant, la prise de position par les autorités de Bamako dans le conflit libyen et les manifestations pro-Kadhafi à Bamako ${ }^{14}$ reflètent les affiliations multiples des migrants maliens. Un rapatrié explique ses engagements politiques ambivalents en fonction de l'évolution de la crise libyenne :

Quand on te prenait avec des images de Kadhafi, c'était dangereux surtout dans des endroits qui n'étaient pas sous contrôle de Kadhafi. Petit à petit, tout le monde a commencé à nous haïr. Mais quand le vent a changé de direction, des personnes qui étaient pour Kadhafi nous incitaient à dire que Kadhafi était « kelb» [chien en arabe]. Ils commençaient à déchirer ses photos ou mettaient la poussière du pied sur celles-ci. On nous demandait de faire autant. Certains me disaient [...] viens mettre tes pieds sur les photos de Kadhafi. Je leur répondais que je suis venu chercher de l'argent, travailler... (entretien avec un rapatrié ayant séjourné pendant 13 ans en Libye, octobre 2015, Bamako)

En définitive, on peut se rendre compte à partir de ces témoignages de I'instrumentalisation des travailleurs migrants pour afficher des prises de position politiques de leurs employeurs : vivant à Tripoli, au début du conflit, l'auteur de cet entretien a dû aller en prison pour avoir nettoyé les vitres de son entreprise avec le

${ }^{14}$ Voir Le Challenger du 29 mars 2011. 
quotidien national sur lequel se trouvait une photo à effigie de Kadhafi. À cette période, le culte voué à Kadhafi était à son paroxysme. Par la suite, quand Tripoli fut pris, ses employeurs lui demandèrent de traiter le "Guide " de kelb. Comme le décrit la situation ci-dessus, l'instrumentalisation de certains migrants dans la crise libyenne fluctuait selon l'évolution du conflit et des positions affichées ou soupçonnées entretenues par les régimes politiques de leur pays d'origine avec le régime de Kadhafi ou de l'opposition (Puig, 2019).

\section{Politisation du retour et construction d'identités plurielles}

L'analyse des relations entre les rapatriés et l'État malien présentée ici se base sur le cas de l'AMARLIM. Cette association distingue la catégorie de rapatriés ayant regagné Bamako via l'action conjointe de l'État malien et de l'OIM. À côté de cette catégorie, existe celle des rapatriés ayant regagné Kidal sans l'intervention de l'État et qui sont présentés comme d'anciens combattants revenus au pays en étant armés. Les membres de I'AMARLIM avaient de multiples attentes vis-à-vis de l'État malien, comme la fourniture d'emplois ou des stratégies pour tirer profit de l'économie de guerre en Libye. Mais, ils aspiraient aussi à plus de justice sociale et à un traitement plus égalitaire de la part des pouvoirs publics maliens. À leur retour au Mali, leurs principaux griefs contre les pouvoirs publics du pays étaient le manque de dispositifs de réinsertion et " une forme supposée de favoritisme " du régime d'ATT à " l'égard des migrants d'origine targui revenus de Libye » qu'ils qualifièrent de politique à « double vitesse de l'État " (Tounkara, $2013: 47$ ). Un responsable de l'AMARLIM fustigeait ainsi l'attitude du régime d'ATT en ces termes: "L'État est aux petits soins des rapatriés touareg, nous les autres, sommes-nous des cochons? » (entretien avec le secrétaire administratif de I'AMARLIM, septembre 2012, Bamako). L'AMARLIM accusait l'État du Mali d'avoir créé une distinction en accueillant des rapatriés d'origine touareg à Koulouba (le palais présidentiel), en envoyant des vivres et de l'argent à certains à Kidal, alors que ceux qui sont retournés à Bamako "trimaient depuis [...] des mois " (Théra, 2011). Des photocopies de laissez-passer des rapatriés touareg sont exhibés par des responsables de l'AMARLIM pour donner de la consistance à leurs accusations de " collusion d'État avec le groupe de rapatriés touareg " (Théra, 2011; Tounkara, $2013: 47$ ).

Rappelons que le retour des militaires libyens de nationalité malienne à Kidal (les rapatriés armés) (Tounkara, 2013) a suscité de vives polémiques au sein de la société civile malienne et fut présenté comme une forme de laxisme du régime d'ATT pour ne pas les avoir désarmés à la frontière :

$\mathrm{Si}$, au départ, le MNLA [Mouvement national de libération de l'Azawad] était ouvert au dialogue avec le pouvoir central et ses actions étaient pacifiques, il disposait d'une frange militaire composée d'hommes d'expérience, des militaires déserteurs de l'armée régulière et des combattants ayant servi en Libye : ces derniers étaient rentrés au pays lourdement armés et bien entraînés. (Grégoire, $2013: 7$ )

Cette attitude du gouvernement malien fut jugée imprudente par I'AMARLIM et d'autres organisations de la société civile bamakoise, alors que l'État malien faisait face 
aux conflits avec des groupes insurrectionnels, à l'effondrement du Nord et au coup d'État du 22 mars 2012. Selon un rapatrié, il aurait mieux valu suivre cette maxime selon laquelle : "Celui dont le père fut tué par un taureau rouge ne devrait-il pas se méfier d'une termitière rouge ? " (entretien avec un rapatrié de la Libye, décembre 2015, Kita). Cette maxime conseille la méfiance et la prudence pour des faits semblables ${ }^{15}$ ou des faits qui se répètent en s'appuyant sur l'analogie du taureau rouge (la première menace : le retour des légionnaires de Libye et leur participation à la rébellion de 1990) et de la termitière rouge (la répétition : le retour des rapatriés armés à Kidal en 2011). Or, pour des rapatriés regroupés au sein de l'AMARLIM, cet accueil avec honneur du gouvernement malien aux rapatriés armés à Kidal, avec les audiences qui leur furent accordées par le président ATT, fut vécu comme une forme de marginalisation des rapatriés membres de I'AMARLIM ; et il a nourri les récriminations du secrétaire à l'information de l'AMARLIM au sujet de leurs conditions d'accueil :

Des gens nous ont accueillis au nom du gouvernement. Ils n'étaient ni des ministres, ni des directeurs. Ils nous ont salués, puis ils sont repartis. Ils nous ont laissés là-bas comme des falatow [orphelins en bamanankan]. On dirait comme un orphelin, tu ne sais pas où aller, dans ton pays, tu deviens comme un misikinè [misérable]. (entretien avec le secrétaire à l'information de l'AMARLIM, décembre 2013, Bamako)

La presse relaya également ce traitement différencié au sein des rapatriés :

Pourtant, nous sommes venus avant ces touareg. Nous n'avons rien reçu de la part du gouvernement alors que nous sommes plus nombreux que ces derniers! (Coulibaly, 2011)

Ces rapatriés de l'AMARLIM se perçoivent marginalisés, comme des laissés-pour-compte de la république, des orphelins et des indigents, dans la mesure où ils trouvent que l'État a été injuste envers ses administrés dans la redistribution de ressources, de justice et d'assistance sociale :

Après les problèmes des arrivées, les Tamasheq étaient escortés jusqu'au palais présidentiel pendant qu'ils nous amenaient dans la cour de la foire d'exposition de Bamako (FEBAK). Lorsque nous avons revendiqué nos droits, les "Blancs ", au nombre de 300 , ont reçu 50 millions de la part du gouvernement. Les Noirs, qui étaient plus de 13000 , n'ont reçu que 500000 francs CFA. Tout le Mali est au courant de cela. Un aventurier noir de la Libye ne vaut rien aux yeux du gouvernement malien. (entretien avec une rapatriée ayant séjourné huit ans en Libye et membre fondatrice de I'AMARLIM, février 2013, Bamako)

Si telle était la position de l'AMARLIM sur l'accueil, les " petits soins de l'État » (entretien avec le secrétaire administratif de l'AMARLIM, septembre 2012, Bamako) ont permis aux rapatriés armés de Libye de prêter allégeance au Mali à cause " de l'hospitalité "symbolique" offerte à ces groupes de rapatriés armés et la visite de six ministres maliens

15 Julien Brachet (2009), André Bourgeot (1990: 143-159) et Pierre Boilley (2011: 4) analysent entre autres le retour des émigrés touareg de Libye comme l'un des éléments participant aux rébellions des années 1990 au Mali et au Niger. 
à Kidal et à Gao en octobre 2011 » (discussion avec un enseignant-chercheur, août 2014, Bamako).

\section{Conclusion}

L'externalisation des frontières de I'UE, les expulsions, l'instrumentalisation des migrations et la subalternisation des emplois, qui sont abordées ici, ont toujours existé dans les migrations contemporaines des migrants subsahariens en Libye.

L'historicité des pratiques migratoires entre le Mali et la Libye est antérieure à la politique d'externalisation des frontières européennes en Afrique et à la légitimation de la criminalisation de la migration irrégulière. En revanche, la gestion contemporaine des migrants subsahariens en Libye est l'héritage d'une pratique bureaucratique qui se justifiait moins par une rationalité sécuritaire propre à l'État libyen, que par sa volonté $d^{\prime}$ 'user du dividende migratoire dans ses relations interrégionales avec ses voisins. Cette utilisation particulière du dividende migratoire par la Libye avait pour objectif de lui donner une nouvelle visibilité dans ses relations diplomatiques avec l'Europe. Au fil du temps, il y a une forte mutation dans la politique libyenne de gestion de l'étranger, d'un accueil économique plutôt neutre, vers un accueil opportuniste fortement politisé, avec des positionnements plutôt violents.

À partir du conflit libyen de 2011, on a assisté à la production du rapatrié qui supplée celle de l'expulsé en cours depuis la seconde moitié des années 1980. Bien que les pouvoirs politiques du Mali n'aient pas répondu favorablement en 2011 à la demande libyenne de réactiver l'accord de l'emploi de la main-d'œuvre malienne datant de 1980, il est dit que des Maliens ont été recrutés en Libye par le biais de voies officieuses : aussi bien au Mali qu'en Libye, dans le rang des travailleurs immigrés comme dans celui des mercenaires, pour servir aux côtés des forces du régime soutenant les partisans de Kadhafi. Ces tentatives auront non seulement de lourdes répercussions sur la présence des immigrés dans la période post-Kadhafi mais aussi dans la gestion du retour des rapatriés au Mali.

\section{Bibliographie}

Adepoju A., 2009, « Migration Management in West Africa within the context of ECOWAS Protocol on Free Movement of Persons and the Common Approach on Migration: Challenges and Prospects ", in M. Trémolières (ed.), Regional Challenges of West African Migration: African and European Perspectives, Paris, OECD Publishing, pp. 17-47.

Agier M., 2008, Gérer les indésirables. Des camps de réfugiés au gouvernement humanitaire, Paris, Éditions Flammarion.

Amnesty International, 2017, « Libye : un obscur réseau de complicités. Violences contre les réfugiés et les migrants qui cherchent à se rendre en Europe ", rapport, 74 p., multigr.

Andersson R., 2014, Illegality, INC. Clandestine migration and the business of bordering Europe, Oakland, California, University of California Press. 
Ballo M., 2009, "Migration au Mali : Profil national », rapport, Genève, OIM, 136 p., https://publications.iom.int/system/files/pdf/mali_profile_2009.pdf (consulté le 30 avril 2020).

Bartolomeo A.D., Jaulin T., Perrin D., 2011, « Le cadre démographique-économique de la migration. Le cadre juridique de la migration. Le cadre socio-politique de la migration », rapport, CARIMProfil migratoire Niger, European University Institute, 11 p., multigr.

Bava S., Boissevain K., 2014, « Dieu, les migrants et les États. Nouvelles productions religieuses de la migration », Année du Maghreb, n 1, pp. 7-15.

Beaugé G., Burgat F., 1986, "La question des migrations en Libye », Maghreb-Machrek, n¹12, pp. 56-68.

Bensaâd A., 2002, "La grande migration africaine à travers le Sahara ", Méditerranée, n 3-4, pp. 4152.

Bensaâd A., 2012, "L'immigration en Libye : une ressource et la diversité de ses usages ", Politique africaine, $\mathrm{n}^{\circ} 125(1)$, pp. 83-103.

Boilley P., 2011, " Géopolitique africaine et rébellions touarègues. Approches locales, approches globales (1960-2011) ", L’Année du Maghreb, nº 7, pp. 151-162.

Bourgeot A., 1990, "Identité touarègue : de l'aristocratie à la révolution », Études rurales, n 120, pp. 129-162

Brachet J., 2009, Migrations transsahariennes vers un désert cosmopolite et morcelé (Niger), Broissieux, Éditions du Croquant.

Brachet J., Choplin A., Pliez O., 2011, " Le Sahara entre espace de circulation et frontière migratoire de l'Europe ", Hérodote, $n^{\circ} 142$, pp. 163-182.

Bredeloup S., Pliez O., 2005, Migrations entre les deux rives du Sahara, Paris, IRD éditions, Armand Colin.

Bredeloup S., Zongo M., 2005, "Quand les frères burkinabè de la petite Jamahiriyya s'arrêtent à Tripoli », Autrepart, n³6, pp. 123-147.

Coulibaly S., 2011, "Pour traitement inégal par rapport aux Maliens de l'armée libyenne : les rapatriés (civils) de la Libye s'indignent ", Afribone, https://afribone.com/?Kidal-Retour-descombattants (consulté le 13 mai 2020).

De Genova N., 2017, The borders of "Europe ». Autonomy of Migration, Tactics of Bordering, Durham and London, Duke University Press.

De Haas H., 2007, "The myth of invasion. Irregular migration from West Africa to the Maghreb and the European Union ", research report, IMI, 79 p., multigr.

Dereims A., 2011, "Le piège ", ou l'errance sans fin des migrants africains, film documentaire, France 5.

Diakité A., 2011, "Expulsion des Maliens de la Libye: La rançon des pétrodollars de Kadhafi », L'Indicateur du Renouveau, https://www.afribone.com/?Expulsion-des-Maliens-de-la-Libye (consulté 30 avril 2020).

Diarra L., 2008, «Expulsion des Maliens de Libye : I'ambassadeur libyen au Mali s'explique ", Le soir de Bamako, http://malijet.com/a_la_une_du_mali/8575-expulsion_des_maliens_de_libye_ I_ambassadeur_libyen_au_mali_s_ex.html (consulté le 30 avril 2020). 
El Miri M., 2018, "Devenir "noir" sur les routes migratoires. Racialisation des migrants subsahariens et racisme global », Sociologie et Sociétés, n², pp. 101-124.

Grégoire E., 2013, "Islamistes et rebelles touaregs maliens: alliances, rivalités et ruptures », EchoGéo, https://journals.openedition.org/echogeo/13466?lang=en (consulté le 24 mai 2020).

Grégoire E., Schmitz J., 2000, "Monde arabe et Afrique noire : permanences et nouveaux liens », Cahiers des sciences humaines, $\mathrm{n}^{\circ} 6, \mathrm{pp}$. 5-20.

Haddad S., 2007, «Anciens et nouveaux parias. Des usages des migrations et du transit dans la politique libyenne ", Revue des mondes musulmans et de la Méditerranée, n 119-120, pp. 2338.

Hamood S., 2006, "African transit migration through Libya to Europe: the human cost ", rapport, Le Caire, The American University, $86 \mathrm{p}$.

Jamana : revue culturelle malienne, 1987, "Les émigrés maliens. Quel destin ? ", n 14-15, numéro spécial, $69 \mathrm{p}$.

Kanté M., Camara A., 1987, " Les travailleurs maliens en Libye ", Jamana : revue culturelle malienne, $n^{\circ} 14-15$, pp. $16-21$

Keita S., 2009, "Migrations internationales et mobilisation des ressources: les Maliens de l'extérieur et la problématique du développement ", in V. Baby-Collin, G. Cortes, L. Faret, H. Guétat-Bernard (dir.), Migrants des Suds, Marseille, IRD Éditions, pp. 217-235.

Le Challenger, 2011, «Le Droit d'en Parler: Discours, déclarations, manifestations pro-Kadhafi : Je m'interroge ", Malijet, http://malijet.com/actualite-politique-au-mali/31463-le_droit_d_en_ parler_discours_d_clarations_manifestations_pro-_k.html (consulté le 26 mars 2020).

Le Tallec C., 2011, "L'arme des mercenaires africains de Kadhafi », La Croix, 3 mars.

Lohmann A., 2011, «Qui sont les Maîtres du Sahara ? Vieux conflits, nouvelles menaces : Le Mali et le Sahara central entre les Touaregs, Al Qaeda et le crime organisé ", rapport, FES, Abuja, 27 p., http://library.fes.de/pdf-files/bueros/nigeria/08231.pdf (consulté le 26 mars 2020).

Lovejoy P., 2011, Transformations in Slavery. A history of slavery in Africa, Cambridge, Cambridge University Press.

Mounkaïla H., 2015, "La gestion des rapatriés de Libye dans la commune de Tchintabaraden (Niger) : les défis de l'urgence et du durable ", Afrique et Développement, $n^{\circ} 1$, pp. 99-117.

Naik A., 2012, "Migrants de retour de la Libye : l'expérience douce-amère du retour au pays ", Genève, OIM, 46 p., multigr.

Perrin D., 2009, "Les migrations en Libye, un instrument de la diplomatie kadhafienne ", OutreTerre, $\mathrm{n}^{\circ} 23$, pp. 289-303.

Pliez O., 2000, "Le Sahara libyen dans les nouvelles configurations migratoires ", REMI, $\mathrm{n}^{\circ} 16$, pp. $165-181$.

Pliez O., 2002, "Vieux réseaux et nouvelles circulations entre les deux rives du Sahara ", Revue Méditerranée, $\mathrm{n}^{\circ}$ 3-4, pp. 31-40.

Pliez O., 2004, "De l'immigration au transit ? La Libye dans l'Espace Migratoire Euro-Africain », in O. Pliez (éd.), La nouvelle Libye, géopolitique, espaces et sociétés au lendemain de l'embargo, Paris, Karthala-Iremam, pp. 130-145. 
Puig O., 2019, « Les migrants nigériens de retour de Libye. Ethnographie entre instabilité, précarité et honnêteté ", E-Migrinter, n 18, pp. 1-13.

Rihouay F., 2012, "Libye : I'Italie passe sous silence un accord "relatif au trafic des migrants" avec le CNT », Jeune Afrique, 22 juin.

Rousseau C., Foxen P., 2006, "Le mythe du réfugié menteur: un mensonge indispensable? », L'évolution psychiatrique, $\mathrm{n}^{\circ} 71$, pp. 505-52.

Scheel S., 2017, "The Secret Is to Look Good on Paper: Appropriating Mobility within and against a Machine of Illegalization ", in N. De Genova (ed.), The borders of "Europe ». Autonomy of Migration, Tactics of Bordering, Durham and London, Duke University Press, pp. 37-63.

Sylla A., 2019, " "C'est devenu si je savais" : les trajectoires de réinsertion des rapatriés maliens de la Côte d'Ivoire et de la Libye entre 2002 et 2017 ", thèse de doctorat en anthropologie, institut de pédagogie universitaire de Bamako, $371 \mathrm{p}$.

Théra K., 2011, "Colère de I'association des Maliens rapatriés de Libye et du Maghreb », L'Indépendant, 28 octobre, https://www.maliweb.net/societe/immigration-emigration/colrede-lassociation-des-maliens-rapatris-de-libye-et-du-maghreb-nous-protestons-contre-laccueilpompeux-rserv-aux-militaires-libyens-dorigine-malienne-alors-que-nous-nous-trimons-depuisnotre-retour-33230.html (consulté le 30 avril 2020).

Tounkara D.-G., 2003, «Quand les migrants demandent la route, Modibo Keita rétorque : "retournez à la terre!" Les "Baragnini" et la désertion du "chantier national" (1958-1968) ", Mande Studies, $\mathrm{n}^{\circ} 5$, pp. 49-64.

Tounkara D.-G., 2013, « La gestion des migrations de retour, un paramètre négligé de la grille $\mathrm{d}^{\prime}$ analyse de la crise malienne ", Politique africaine, $\mathrm{n}^{\circ}$ 130(2), pp. 47-68.

Valluy J., 2006, "Genèse du 'faux-réfugié' », Plein droit, n 69, pp. 19-22. 